\title{
HABERMAS, Rebekka, Diebe vor Gericht. Die Entstehung der modernen Rechtsordnung im 19. Jahrhundert
}

Falk Bretschneider

\section{OpenEdition}

Édition électronique

URL : http://journals.openedition.org/ifha/1859

DOI : 10.4000/ifha.1859

ISSN : 2198-8943

\section{Éditeur}

IFRA - Institut franco-allemand (sciences historiques et sociales)

Référence électronique

Falk Bretschneider, "HABERMAS, Rebekka, Diebe vor Gericht. Die Entstehung der modernen

Rechtsordnung im 19. Jahrhundert », Revue de I'IFHA [En ligne], Date de recension, mis en ligne le 01 janvier 2009, consulté le 22 septembre 2020. URL : http://journals.openedition.org/ifha/1859 ; DOI : https://doi.org/10.4000/ifha.1859

Ce document a été généré automatiquement le 22 septembre 2020.

(CIFHA 


\title{
HABERMAS, Rebekka, Diebe vor Gericht. Die Entstehung der modernen Rechtsordnung im 19. Jahrhundert
}

\author{
Falk Bretschneider
}

1 À l'origine de l'État de droit moderne se trouve le petit larcin ou, plus précisément, la quantité impressionnante de procès pour infractions contre la propriété qu'a connue le XIXe s. C'est à cette observation que s'attache R.H. dans une magnifique étude qui, centrée sur la région de Marbourg en Hesse, part d'une question à première vue très simple : comment expliquer l'intérêt immense qu'ont alors porté la justice, mais également les criminologues, les écrivains ou les journalistes, à des délits mineurs dont l'objet était rarement davantage qu'une bouchée de pain ou un chapeau chiffonné ? L'historiographie s'interroge depuis longtemps sur ce phénomène en y apportant des réponses qui l'inscrivent, généralement, dans une perspective de lutte des classes. L'approche de R.H. est toute autre. S'inspirant de l'anthropologie du droit, elle voit dans cette obsession des contemporains pour le vol la conséquence d'un changement à la fois des rapports à la propriété et de l'ordre juridique. Rien de nouveau, dira-t-on. Certes, au fond, elle ne bouleverse pas les choses. Mais dans les détails, ce livre nous propose une vision entièrement renouvelée. Pour R.H., l'évolution des normes et des pratiques de la justice ne saurait être expliquée par les modèles traditionnels de l'histoire du droit ou de l'histoire sociale; elles ne résultent ni de l'action de quelques grands juristes, ni des seuls conflits entre les classes sociales, mais émanent plutôt d'un processus performatif qui est déterminé par de multiples interactions entre les acteurs sociaux (p. 20). C'est à ce processus que l'auteure s'intéresse, avec des résultats parfois étonnants.

Dans la première partie du livre, R.H. s'interroge sur les origines des petits voleurs, sur leurs victimes et sur les processus qui amènent un délit devant le tribunal. De ses observations résulte vite un constat curieux : le vol, au XIXe s., est un phénomène des couches inférieures - des deux côtés du crime. Les voleurs sont généralement des journaliers jeunes, masculins et célibataires. Leurs victimes, quant à elles, sont 
fréquemment de «bonnes vieilles connaissances » (p. 36), c'est-à-dire des gens issus du même village et dont la situation socio-économique ne diffère guère. Le crime se passe à l'intérieur d'une société paysanne marquée par une existence à la lisière de la misère et dans laquelle, cependant, de nombreuses petites différences économiques, confessionnelles, sociales ou juridiques existent. C'est le durcissement de ces différences, à la suite des réformes réduisant un tissu complexe de stratégies traditionnelles de survie au seul rapport entre possédants et non possédants, qui rend de plus en plus précaire la situation des uns et les amène à commettre des larcins chez les autres. Les mobiles du vol ne se résument pourtant pas à l'indigence (de même que les voleurs ne peuvent être ramenés à la seule figure, chère à l'histoire sociale traditionnelle, du " rebelle social »). Les objets volés revêtent plutôt une dimension hautement symbolique : à nos yeux, ce sont des objets sans valeur; pour les contemporains, en revanche, ils représentent le cœur même de leur existence. Pain, haricots ou pommes de terre fournissent la nourriture quotidienne, chemises, chapeaux ou bottes forment des objets intimes se référant à l'identité personnelle (p. 57). C'est donc en leur qualité de marqueurs sociaux que l'on peut comprendre, selon R.H., pourquoi de telles choses ont pu devenir l'objet du désir des criminels aussi bien que de leurs victimes (qui, souvent, se sont efforcées avec ardeur de les récupérer). Mais pour quelle raison ces conflits quotidiens, qui sévissaient déjà bel et bien sous l'Ancien Régime, débouchent-ils au XIXe s. sur une action judiciaire ? Pour l'auteure, le recours au tribunal que pratiquent tous ces humbles, soucieux de rentrer en possession d'un objet apparemment banal, exprime le besoin de rétablir, sous le regard de la communauté, leur honneur bafoué par le crime. Prenant ainsi le relais des innombrables procès pour injures qui ont marqué l'époque moderne, ces plaintes pour vol résultent toutefois d'un " problème de traduction » (p. 74) : alors que les plaignants mettent souvent un conflit quotidien (dans toute sa complexité sociale) entre les mains de la police, les agents de celle-ci n'y voient, dans le contexte de la redéfinition juridique de la propriété, qu'une affaire de vol. L'« optique juridique » (p. 86) efface alors tous les autres enjeux $d u$ conflit, transformant ainsi une réalité sociale embrouillée en une simple infraction contre la propriété.

Cette réduction de la complexité du jeu social se poursuit lors de l'instruction. Dans la deuxième partie de son ouvrage, R.H. montre à quel point la constitution de la preuve (recours au casier judiciaire, à des certificats de bonnes mœurs ou à des avis de recherche, perquisition à domicile, inspection méticuleuse du lieu du crime...), en se pliant à l'impératif de l'objectivité et en s'inscrivant de ce fait dans le surgissement de nouvelles technologies de la vérité (statistique, casuistique juridique, criminologie) propres au XIXe s., contribue non seulement à décontextualiser, à désubjectiviser et à quantifier le délit, mais aussi à réduire son auteur à une créature sans relations sociales ni intérêts propres (p. 129). Dans cette même perspective se place l'interrogatoire, concentré ostensiblement sur la valeur matérielle des biens volés, si bien qu'il masque toute autre question apparemment insignifiante sur le contexte social du crime et qu'il solidifie de surcroît, petit à petit, la notion moderne de propriété (p. 151), seule vérité qu'il faille attendre désormais des procédés juridiques.

4 L'audience devant le tribunal enfin, qui fait l'objet de la troisième partie du livre, ne représente guère, selon R.H., le symbole d'une réforme visant à garantir l'égalité des droits, la justice et le contrôle de l'État par la société. À l'encontre de ces interprétations traditionnelles, l'auteure constate que la majorité des procès pour vol n'avaient de toute façon pas lieu devant les assises (incarnation d'un ordre juridique 
moderne) et qu'en outre les débats publics se contentaient généralement de reprendre les résultats déjà obtenus lors de l'instruction. L'« illusion » (p. 181) du contrôle de la justice par l'espace public est pourtant essentielle à la légitimité du tribunal en tant que lieu central et unique de la naissance du droit ; elle engendre donc une série de symboles (palais de justice monumentaux), de rituels collectifs (lever du public lors des prestations de serment) et de règles rhétoriques destinés à conférer les apparences de la dignité et de l'importance. Néanmoins, non seulement nous ne savons pas grandchose sur la présence réelle du public, mais il semble également que les débats se soient déroulés généralement sous la seule régie des juges, les jurés n'exerçant que rarement leurs droits et les avocats étant surmenés, mal payés et engagés dans des relations d'interdépendance avec leurs collègues à l'autre bout du prétoire. Cependant, l'audience n'est pas une farce servant seulement à la fixation d'interprétations déjà établies (p. 210). Elle crée des dynamiques imprévues (témoins ne se résignant pas au rôle qu'on leur assigne) et fait également l'objet d'une constante réinterprétation dans la presse locale où, contrairement à la logique juridique, l'accent n'est pas mis sur la liturgie du code de la procédure pénale, mais sur les accidents de la vie quotidienne se cachant derrière le sort de ces petits voyous condamnés à la chaîne. C'est par la presse, pourrait-on conclure des fines analyses que R.H. nous livre dans ces chapitres, que le quotidien se réintroduit donc dans la sphère judiciaire, et dans les faits divers resurgit un spectacle de la transgression apposant au monde austère et exsangue du droit un univers fait d'ambiguïtés, d'imprévisibilités et de disparités, bref : la vie.

5 Au XIXe s., le tribunal devient ainsi un « lieu paradoxal » (p. 234) où de l'amalgame de nombreuses contradictions naît un nouvel ordre juridique. Ses accoucheurs ne se réduisent pas aux seuls juristes ; bien au contraire, le droit est devenu une affaire publique, et l'intérêt à première vue démesuré que presque tous les contemporains ont porté au vol et à son traitement par la justice n'en est pas l'indice le moins probant. Avoir décrit avec perspicacité et une grande finesse ces transformations, tel est le mérite d'un livre qui, sans aucun doute, fera date dans l'historiographie allemande de la criminalité et de la justice pénale - et au-delà.

6 Falk BRETSCHNEIDER (École des Hautes Études en Sciences Sociales, Paris) 